\title{
Study on the Optimal Equivalent Radius in Calculating the Heat Dissipation of Surrounding Rock
}

\author{
H. T. Song ${ }^{1, *}$, Y. P. Qin ${ }^{1}$, J. S. Wu ${ }^{1}$, S. Kong ${ }^{1}$, Z. W. Wang ${ }^{1}$ and R. Leng ${ }^{2}$ \\ ${ }^{1}$ Faculty of Resources and Safety Engineering, China University of Mining and Technology (Beijing), Beijing 100083, China \\ ${ }^{2}$ Division of Safety and Production Engineering, UPC, G.ue Tecch 11, Naples, Italy
}

Received 18 June 2015; Accepted 17 November 2015

\begin{abstract}
The heat dissipation of surrounding rock of a non-circular roadway is computed using an equivalent circular roadway approach under three circumstances when the area, perimeter, or hydraulic diameter of the circular roadway is equal to the non-circular roadway to obtain the optimal equivalent radius. The differential equations of heat conduction for unstable surrounding rock are established in cylindrical and rectangular coordinate systems using dimensionless analysis method. The calculation formulas of heat dissipation capacity and heat transfer resistance are derived from differential equations. Based on the method of equivalent radius, the similarities and differences between non-circular and circular roadways in calculating the heat dissipation of surrounding rock are discussed. Using the finite volume method, the calculation models for non-circular and circular roadways in the heat dissipation of surrounding rock are also established, among the non-circular roadways including three circumstances, namely, trapezoid, rectangle, and arch. The relation errors of heat dissipation of the surrounding rock of the three equivalent circular roadway methods are investigated for the three non-circular roadways. Results show that the calculation approach with equal perimeters is the best for the heat dissipation of surrounding rock of non-circular roadways.
\end{abstract}

Keywords: Non-circular Roadway, Circular Roadway, Heat Dissipation of Surrounding Rock, Equivalent Radius

\section{Introduction}

With the increase in mining depth, a high-temperature geothermal field has become a serious problem in mine in recent years. In South Africa, Bluhm et al. reported that the mining depth of the working face on Witwatersrand reached $3800 \mathrm{~m}$ where virgin rock temperature exceeded $60{ }^{\circ} \mathrm{C}[1]$. Meanwhile, based on related statistics in China, 47 mines have mining depth of over $1000 \mathrm{~m}$ and the working face temperature of approximately $36{ }^{\circ} \mathrm{C}$ [2]. Three different modes of deep mine geothermal field, namely, linear, nonlinear, and abnormal, were also previously presented [3]. Yuan analyzed the causes of formation of heat hazards in Huainan and provided some suggestions in prevent heat damage [4]. Based on the influence of thermal hazard in deep mine, Yang et al. summarized the causes of deep thermal hazard and compared the control technologies for deep thermal hazards [5]. According to cooling systems consume up to $25 \%$ of the total electricity, Gideon et al. proposed an energy saving strategy on a deep mine cooling system [6]. In [7], some measures and solutions were presented by analysis the environmental issues from coal mining. It's found that the high temperature condition can severely impair the health of coal miners and can evoke several disastrous incidents, such as fire and gas explosion [8]. Therefore, a study on the characteristics of the geothermal field and the control of heat hazards is

* E-mail address: songhuaitao8803@163.com ISSN: 1791-2377 (C) 2015 Kavala Institute of Technology. All rights reserved. significant in improve coal miners' working condition.

In this study, we mainly focus on discuss the influence of sectional form of roadways in calculate the heat dissipation of surrounding rock between non-circular and circular roadways. The heat quantity from rock around roadways is one of the main heat resources in a high-temperature mine, and its heat dissipation capability exhibits a close relationship with the sectional form of roadways, except for the thermo-physical properties of rocks, airflow temperature, wind speed, wall humidity and ventilation time [9], [10]. Based on different cross-sectional shapes, roadways can be divided into two types, namely, non-circular and circular roadways. A circular roadway possesses symmetry. Consequently, the calculation of heat dissipation capability using an analytical solution is relatively simple. However, most roadways in mines are non-circular in addition to the shaft, which is relatively complex. As such, obtaining its analytical solution is difficult. Therefore, an equivalent circular roadway approach is commonly employed for noncircular roadways in calculate the heat dissipation of surrounding rock. However, the specific length of the equivalent circular roadway, i.e., the so-called equivalent radius, has not yet been unified. Three well-known methods [11], namely, (1) equal area, (2) equal perimeter, and (3) equal hydraulic diameter methods, have been used in calculate the heat dissipation of surrounding rock of the noncircular roadway. Therefore, the application of the equivalent circular radius is extremely confusing in computing the heat dissipation of surrounding rock.

Many studies on calculating heat quantity from rock around roadways have been reported. Щсрбаиъ.АП [12] 
proposed an unstable heat transfer criterion, which had a relationship with the heat dissipation of surrounding rock and was influenced by the Fourier and Biot numbers. Cen et al. proposed an analytical solution for the unstable heat transfer criterion using the variable separation approach [13] However, the calculation method for the practical application was complex and difficult. Sun et al. [14] simplified the problem using the Laplace transformation approach and provided an analytic expression of the unsteady heat transfer coefficient on the basis of the equivalent circular roadway model to remedy the deficiencies of the solution of Cen et al. Starfield et al. proposed a theoretical approach to describe heat dissipation between surrounding rock and airflow under a moist condition [15]. In [16], Yakovenko et al. analyzed the heat transfer coefficient between airflow and surrounding rock with a small Fourier number. With regard to the deficiencies of the methods of Starfield et al. and Yakovenko et al., Gao et al. analyzed the influence of moisture on the heat dissipation of surrounding rock using the equal area method [17]. They also proposed a theory that heat released from surrounding rock was greater and airflow temperature was higher compared with water vaporized on the airway surface when water evaporation occurred during airflow. However, the shape of the roadway was not considered. In [18], Zhang et al. analyzed the coupled problem of heat transfer in the surrounding rock and heat convection between the air and the surrounding rock in the Fenghuo mountain tunnel. Considering the characteristics of the temperature field, such as an unsteady state, heterogeneous rocks and anisotropy, and anomalous forms of laneways, $\mathrm{Wu}$ et al. proposed a theoretical approach based on the finite element method and analyzed the basic law of the change of the surrounding rock temperature field with time and space [19], [20]. Lowndes et al. [21] built a set of experimental devices to simulate the convective heat transfer coefficient and obtained the change relationship between the wall temperature of surrounding rock and flow temperature. In [22], Zhang proposed a mathematical model for the temperature field established under cylindrical coordinates to verify the feasibility of the modeling experimental method, which was a supplement to the model of Lowndes. Based on CLIMSIM and MULTIFLUX, Danko et al. [23] investigated the transfer phenomena in heat and mass on a roadway wall. Considering that the flow temperature periodically changed with time, Qin et al. determined that the fluctuation amplitude of temperature presented a negative exponential change with the increase in depth of surrounding rocks using the equal area method [24]. To date, only a few studies have been conducted on the effect of the sectional form of roadways on the heat dissipation of surrounding rock.

In practice, in order to simplify calculation, equivalent methods used to measure a circular roadway can be applied to measure non-circular roadways. According to the above investigations, each equivalent method exhibits a similar relationship curve for the unstable heat transfer criterion $(\mathrm{Ku} \tau)$ with Fourier and Biot numbers, as shown in Figure 1. However, every equivalent method has different Fourier and Biot numbers, which results in varying heat dissipation levels for a practical problem. The reasons that the different result is discussed in calculate the heat dissipation of surrounding rock using the different equivalent circular roadway approaches.

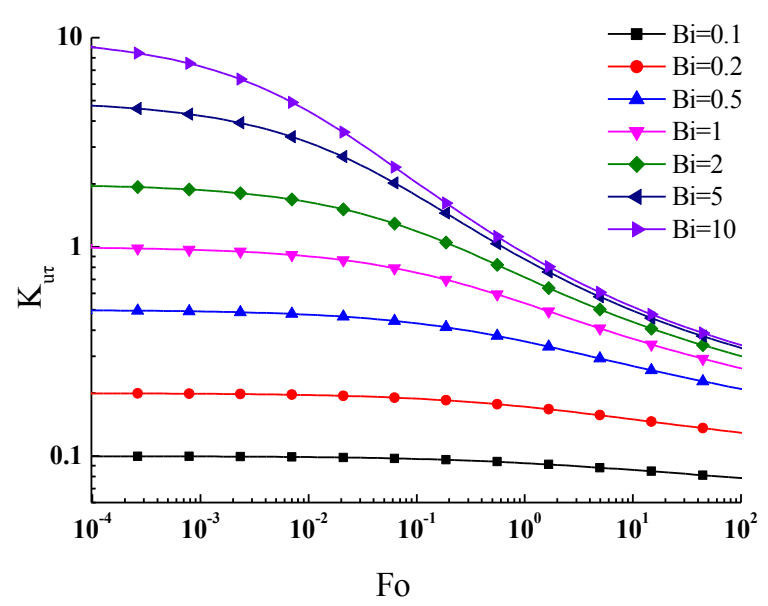

Fig. 1. The relationship curve for the unstable heat transfer criterion with Fourier and Biot numbers

In this study, the heat dissipation of surrounding rock of a non-circular roadway is computed using the equivalent circular roadway approach under three circumstances when the area, perimeter, or hydraulic diameter of the equivalent circular roadway is equal to those of the non-circular roadway to obtain the optimal equivalent radius. Noncircular roadways with trapezoidal, rectangular, and arch cross-sectional forms are shown in Figure 2. Based on theory of equivalent method, the similarities and differences of surrounding rock heat dissipation of non-circular and circular roadways are analyzed. Ultimately, the deviations of surrounding rock heat dissipation of equivalent circular roadways are investigated under the three trapezoidal, rectangular, and arch cross-sectional forms.



(a) Trapezoid Roadway

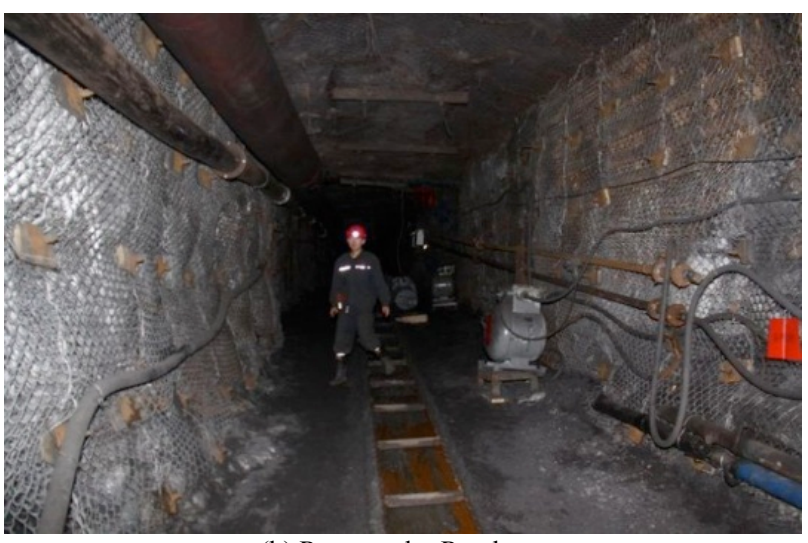

(b) Rectangular Roadway 


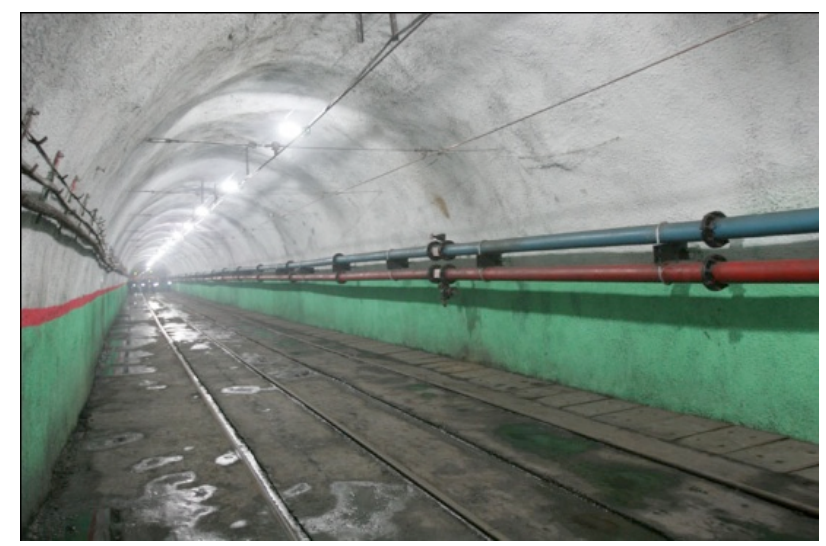

(c) Arch Roadway

Fig. 2. Non-circular roadways in mine

\section{Methodology}

\subsection{Established Governing Equations of Thermal} Conductivity of Surrounding Rock

The circular roadway is analyzed in the cylindrical coordinate system and the non-circular roadway is analyzed in the rectangular coordinate system to simplify the calculations. Meanwhile, several necessary assumptions are as follows. (1) Only the heat flow of the radial direction is considered, whereas that of the axial direction is ignored. (2) Sensible heat and latent heat on the surface of surrounding rocks are not considered. (3) The surrounding rock is homogeneous and isotropic. Based on the law of conservation of energy and Fourier's law, the governing equations of thermal conductivity for the evolution of the temperature field are given as follows:

$$
\left\{\begin{array}{l}
\frac{\partial T^{2}}{\partial x^{2}}+\frac{\partial T^{2}}{\partial y^{2}}=\frac{1}{a} \frac{\partial T}{\partial t} \\
\frac{\partial^{2} T}{\partial r^{2}}+\frac{1}{r} \frac{\partial T}{\partial r}=\frac{1}{a} \frac{\partial T}{\partial t} \\
\left.T\right|_{t=0}=T_{g u} \\
-\left.\lambda \frac{\partial T}{\partial n}\right|_{\Gamma 1}=h\left(T_{w}-T_{f}\right) \\
\left.T\right|_{\Gamma 2}=T_{g u}
\end{array}\right.
$$

where, $T$ is the temperature of surrounding rocks; $x$ and $y$ are the values in the rectangular coordinate system; $r$ is the distance from the outside boundary of the roadway to the center in the cylindrical coordinate system; $t$ is the ventilation time; $n$ is the direction of the boundary outer normal line; $a$ is the temperature conductivity of surrounding rocks; $a=\lambda / \rho c, \lambda$ is the thermal conductivity, $\rho$ is the density, $c$ is the specific heat; $h$ is the convective heat transfer coefficient between surrounding rocks and airflow; $T_{\mathrm{gu}}$ is the virgin rock temperature; $T_{\mathrm{w}}$ is the surface temperature of surrounding rocks; $T_{\mathrm{f}}$ is the airflow temperature of the roadway; and $\Gamma_{1}$ and $\Gamma_{2}$ are the internal and external boundaries of surrounding rocks, respectively.

\subsection{Dimensionless Governing Equations}

Dimensionless numbers are integrated into Eq. 1 to derive Eq. 2, as follows:

$$
\left\{\begin{array}{l}
\Theta=\frac{T-T_{a}}{T_{0}-T_{a}} \\
\Theta_{w}=\frac{T_{w}-T_{f}}{T_{g u}-T_{f}} \\
X=\frac{x}{r_{0}} \\
Y=\frac{y}{r_{0}} \\
R=\frac{r}{r_{0}} \\
N=\frac{n}{r_{0}} \\
B i=\frac{h r_{0}}{\lambda} \\
F o=\frac{a t}{r_{0}^{2}}
\end{array}\right.
$$

where, $\Theta$ denotes the dimensionless excess temperature; $\Theta_{\mathrm{w}}$ denotes the dimensionless excess temperature of the surrounding rock surface; $X$ is the dimensionless abscissa; $Y$ is the dimensionless ordinate; $R$ is the dimensionless radius, $N$ is the surface normal direction; $\mathrm{Bi}$ is the Biot number; Fo is the Fourier number; and $r_{0}$ is the equivalent radius of the roadway calculated in Eq. 3, as follows:

$$
\left\{\begin{array}{l}
r_{0,1}=\sqrt{\frac{S}{\pi}} \\
r_{0,2}=\frac{U}{2 \pi} \\
r_{0,3}=\frac{4 S}{U}
\end{array}\right.
$$

where, $r_{0,1}$ is the radius of the equal area method; $r_{0,2}$ is the radius of the equal perimeter method; $r_{0,3}$ is the radius of the equal hydraulic diameter method; $S$ is the cross-sectional area; and $U$ is the cross-sectional perimeter.

By substituting Eq. 2 into Eq. 1, we can obtain Eq. 4, as follows:

$$
\left\{\begin{array}{l}
\frac{\partial^{2} \Theta}{\partial X^{2}}+\frac{\partial^{2} \Theta}{\partial Y^{2}}=\frac{\partial \Theta}{\partial F O} \\
\frac{\partial^{2} \Theta}{\partial R^{2}}+\frac{1}{R} \frac{\partial \Theta}{\partial R}=\frac{\partial \Theta}{\partial F_{O}} \\
\left.\Theta\right|_{F o=0}=1 \\
-\left.\frac{\partial \Theta}{\partial N}\right|_{\Gamma 1}=B i \Theta_{w} \\
\left.\Theta\right|_{\Gamma 2}=1
\end{array}\right.
$$

From Eq. 4, $\Theta$ is the function of $\mathrm{Bi}, \mathrm{Fo}$, and $\mathrm{R}$ in the equivalent circular roadway. Therefore, we can derive Eq. 5, as follows:

$\Theta=f(B i, F o, R)$ 
Meanwhile, the equivalent radius of the roadway is 1 at the surrounding rock surface. By rearranging Eq. $5, \Theta_{\mathrm{w}}$ can be expressed in Eq. 6, as follows:

$\Theta_{w}=f(B i, F o)$

\subsection{Calculating Heat Dissipation Capability}

After the excavation of a roadway, airflow blows across the rock surface and convective heat transfer occurs between airflow and surrounding rocks. Given that the temperature field of surrounding rocks exhibits unsteady heat conductivity, heat flux density based on Newton's law of cooling can be expressed in Eq. 7, as follows:

$$
q=h\left(T_{w}-T_{f}\right)=\frac{B i \lambda}{r_{0}} \Theta_{w}\left(T_{g u}-T_{f}\right)
$$

where, q denotes heat flux density.

From Eq. 7, heat dissipation capacity at unit length can be expressed in Eq. 8, as follows:

$$
Q_{l}=\frac{Q}{l}=\frac{B i \lambda U}{r_{0}} \Theta_{w}\left(T_{g u}-T_{f}\right)
$$

where, $\mathrm{Q}$ is the heat dissipation capability; $\mathrm{Q}_{l}$ is the heat dissipation capability at unit length; and $l$ is the length of the roadway.

Therefore, the unstable heat transfer criterion $\left(\mathrm{K}_{\mathrm{u}}\right)$ [20] can be expressed in Eq. 9, as follows:

$$
K_{u \tau}=B i \Theta_{w}
$$

Based on Eq. 9, the relationship curve of $\mathrm{K}_{\mathrm{u \tau}}, \mathrm{Fo}$, and Bi is shown in Figure 1.

By substituting Eq. 9 into Eq. 8, heat dissipation capability at unit length can be expressed in Eq. 10, as follows:

$$
Q_{l}=K_{u \tau} \frac{\lambda U}{r_{0}}\left(T_{g u}-T_{f}\right)
$$

\subsection{Calculating Heat Transfer Resistance}

In general, the two methods of heat transport between surrounding rock interior and airflow are as follows: heat conduction from the surrounding rock interior to the surrounding rock surface and natural heat convention from the surrounding rock to the flow. That is, the two types of thermal resistances during the thermal transformations are as follows: heat conduction resistance and heat convection resistance. Therefore, the heat quality from rocks around roadways is influenced by heat conduction and heat convection. Using the concept of thermal resistance, the heat quantity between the surrounding rock surface and airflow per unit length can be expressed in Eq. 11, as follows:

$Q_{l}=\frac{T_{g u}-T_{f}}{\beta_{l}}$

where, $\beta_{l}$ is the heat transfer resistance.

From Eq. 8 and 9, the heat transfer resistance $\left(\beta_{l}\right)$ can be expressed in Eq. 12, as follows:
$\beta_{l}=\frac{r_{0}}{B i \Theta_{w} \lambda U}=\frac{r_{0}}{K_{u \tau} \lambda U}$

Therefore, heat transfer resistance is closely related to the heat transfer criterion.

\subsection{Comparison of Heat Dissipation between Circular and Non-circular Roadways}

\subsubsection{Analysis of Heat Dissipation of Surrounding Rock}

The application of the equivalent circular roadway to measure a non-circular roadway should have the same initial conditions to ensure that the same results of the heat dissipation of the surrounding rock are obtained. Otherwise, this equivalent calculation is nonsense. These expressions are shown in Eq. 13 and Eq. 14, as follows:

$\left\{\begin{array}{l}\lambda_{Y}=\lambda_{F} \\ c_{Y}=c_{F} \\ \rho_{Y}=\rho_{F} \\ h_{Y}=h_{F} \\ T_{f, Y}=T_{f, F} \\ T_{g u, Y}=T_{g u, F} \\ t_{Y}=t_{F}\end{array}\right.$
$\left\{\begin{array}{l}\Theta_{w, Y}=\Theta_{w, F} \\ Q_{l, Y}=Q_{l, F}\end{array}\right.$

Where, subscript $\mathrm{Y}$ denotes the physical quantity of the equivalent circular roadway and subscript $\mathrm{F}$ denotes the noncircular roadway.

The analysis of the synthesis of Eq. 11, 12, 13, and 14 can be expressed as follows:

$\beta_{l, Y}=\beta_{l, F}$

The three circumstances where in the area, perimeter, and hydraulic diameter are equal can be expressed in Eq. 16, as follows:

$$
\left\{\begin{array}{l}
r_{0,1, Y}=r_{0,1, F} \\
r_{0,2, Y}=r_{0,2, F} \\
r_{0,3, Y}=r_{0,3, F}
\end{array}\right.
$$

By substituting Eq. 12 into Eq. 15, we can obtain Eq. 17, as follows:

$$
\Theta_{w, Y} U_{Y}=\Theta_{w, F} U_{F}
$$

Therefore, when the equivalent circular roadway has the same perimeter as the noncircular roadway, $\Theta_{w, Y}=\Theta_{w, F}$ can be obtained using Eq. 17. From the above analysis, it is found that the equivalent perimeter between non-circular and circular roadways is the best method for calculating the heat dissipation of surrounding rock.

\subsubsection{Established Physical Model of Surrounding Rock}

Three roadways, namely, trapezoid, rectangle, and arch, are selected to analyze the generality and difference of the three types of equivalent circular roadway methods. Qin et al. proposed a method for the surrounding rock heat dissipation 
of a circular roadway based on the finite volume method in a $1 \mathrm{D}$ coordinate system [25]. The discretization of Eq. 1 in the rectangular coordinate by Wang [26] is adopted in this study.

The closer the surface of the surrounding rock, the more intense the heat dissipation of surrounding rock. Thus, the triangle unit is selected as the basic unit. The closer the roadway surface, the smaller the unit size. The mass generation of surrounding rocks has been evaluated, and the calculation result is grid independent. The radial grid units of the circular and non-circular roadways are divided into 30 parts, with the ratio of 1:3. The loop grid of the noncircular roadway is divided into 36 parts.

To simplify calculation and provide a convenient contrastive analysis for different equivalent circular roadways, the following assumptions are made: the sectional area for each non-circular roadway is $\pi$; the proportion of the upper base, lower base, and height of the isosceles trapezoid roadway is $3: 5: 2.5$; the proportion of the width and height of the rectangle roadway is $5: 3$; the proportion of the width and height of the semicircular arch is 4:3.5; and the calculation depth is 60 . The physical models of different shapes are shown in Figure 3.

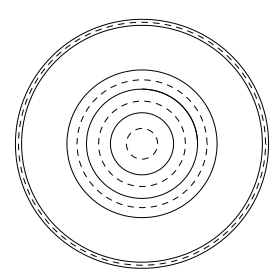

(a) Circular

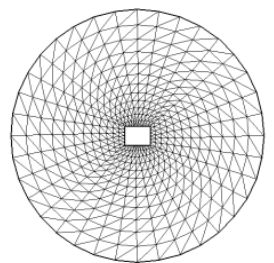

(c) Rectangular

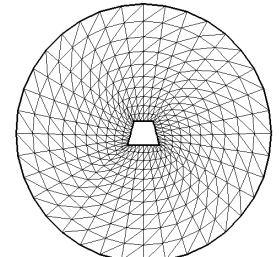

(b) Trapezoid

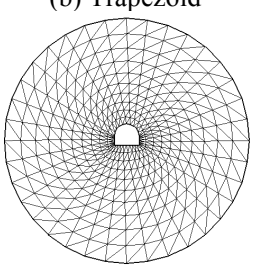

(d) Arch
Fig. 3 Discretization of Different Shape Roadway

\subsubsection{Equivalent Radius of Non-circular Roadways}

For the aforementioned three non-circular roadways, three equivalent circular roadway methods are adopted. Each equivalent radius is shown in Table 1.

Table 1. Different shapes corresponding to three equivalent radii.

\begin{tabular}{cccc}
\hline \multirow{2}{*}{ Equivalent radius methods } & \multicolumn{3}{c}{ Roadway shapes } \\
\cline { 2 - 4 } & Trapezoid & Rectangle & Arch \\
\hline Equal area $\left(r_{0,1}\right)$ & 1 & 1 & 1 \\
Equal perimeter $\left(r_{0,2}\right)$ & 1.285 & 1.165 & 1.069 \\
Equal hydraulic diameter $\left(r_{0,3}\right)$ & 1.557 & 1.716 & 1.870 \\
\hline
\end{tabular}

Based on the definition of Fo and $\mathrm{Bi}$ in Eq. 2, the proportion of three $\mathrm{Bi}$ for the equivalent circular roadway of each shape is $r_{0,1}: r_{0,2}: r_{0,3}$ and the proportion of three $F_{0}$ is $\left(r_{0,1}\right)^{-2}:\left(r_{0,2}\right)^{-2}:\left(r_{0,3}\right)^{-2}$. For example, the proportion of three $\mathrm{Bi}$ for a trapezoid is $1: 1.285$ : 1.557 and the proportion of three Fo is 1: 1.651: 2.423 .

For the non-circular roadway in the rectangle coordinate system, the three equivalent types are essentially equal, even when their Bi and Fo are different. By contrast, for the three equivalent circular roadways, the physical model discrepancy between the circular roadway and the non- circular roadway leads to different results despite the $\mathrm{Bi}$ and Fo of the non-circular roadway being the same as those of the equivalent circular roadway. This study discusses the discrepancy in obtaining the optimal method.

\subsubsection{Analysis of Surface Temperature of Surrounding Rock}

The non-circular roadway is not strictly symmetrical. As such, the border grid units are divided unequally. In particular, the grid in the boundary of corners is more densely divided. Thus, the loop border node temperature is different and is solved using the weighted average method expressed in Eq. 18, as follows:

$\Theta_{w}(F o)=\frac{\frac{\Theta_{1}+\Theta_{2}}{2} L_{1}+\frac{\Theta_{2}+\Theta_{3}}{2} L_{2}+\ldots+\frac{\Theta_{\mathrm{z}}+\Theta_{1}}{2} L_{\mathrm{z}}}{L}$

where, $\mathrm{z}$ is the unit number of the loop grid; $\Theta_{1}, \Theta_{2}, \ldots, \Theta_{z}$ are the loop border node temperatures; and $\mathrm{L}_{1}, \mathrm{~L}_{2}, \ldots, \mathrm{L}_{\mathrm{z}}$ are the lengths of the loop border units.

\subsection{Analysis of Deviation}

To our knowledge, the less the relative error $(\xi)$, the more accurate the result. Thus, three equivalent circular roadway methods can be evaluated using the relative error expressed in Eq. 19:

$$
\xi=\frac{Q_{F}-Q_{Y}}{Q_{F}} \times 100 \%
$$

where, $\mathrm{Q}_{\mathrm{F}}$ is the heat dissipation capacity of the non-circular roadway; $Q_{Y}$ is the heat dissipation capacity of the equivalent circular roadway.

To simplify the calculation, Eq. 10 is substituted into Eq. 19 to obtain Eq. 20, as follows:

$$
\xi=\frac{K_{u \tau, F} U_{F}-K_{u \tau, Y} U_{Y}}{K_{u \tau, F} U_{F}} \times 100 \%
$$

Based on Table 1, the proportion of $\mathrm{Bi}$ and Fo for the three equivalent circular roadways for the same problem can be obtained. Then, based on the ratio relationship, the relative error of the three methods can be compared using Eq. 20.

\section{Results}

The heat dissipation of surrounding rock of non-circular roadways is computed under three circumstances when area, perimeter, or hydraulic diameter is equal to obtain the optimal equivalent radius. Meanwhile, we employ Fourier time as the abscissa to simplify the problem. On the one hand, comparing the results of circular and non-circular roadways is convenient. On the other hand, analyzing the deviation exhibits universality. Based on Eq. 20 and the initial condition, the surrounding rock heat dissipation of the aforementioned three roadways is computed using the finite volume method in the rectangular coordinate system, in which roadway surface temperature changes with time. From Eq. 9, we can obtain the unsteady state heat transfer criterion of the non-circular roadway at any Fourier number. 
Meanwhile, for any equivalent circular roadway, the unsteady state heat transfer criterion at any Fourier number is also obtained based on the foundation model of Qin et al. [25]. Finally, the aforementioned results are successively substituted into Eq. 20, in which the relative error of the three non-circular roadways can be obtained under three circumstances when area, perimeter, or hydraulic diameter is equal, as shown in Figures 4, 5, and 6.

\subsection{Analysis of Relative Errors of Trapezoid Roadway}

(a) Equal area approach

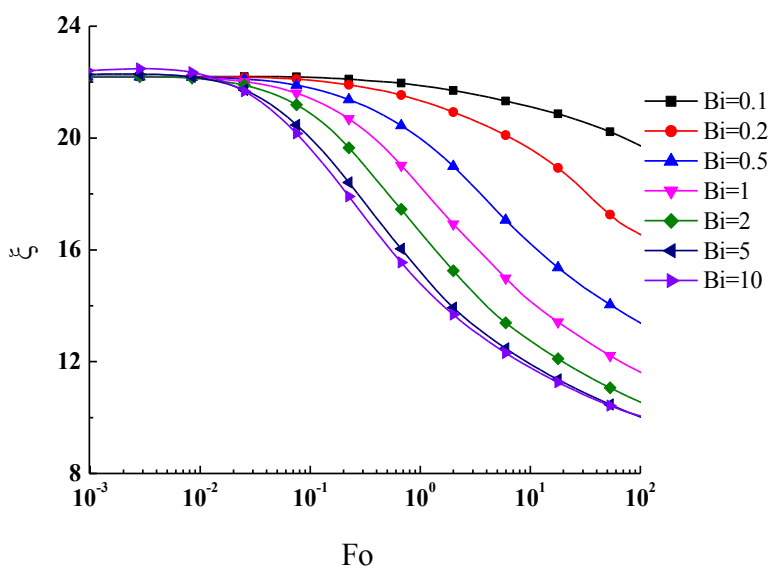

(b) Equal hydraulic diameter approach

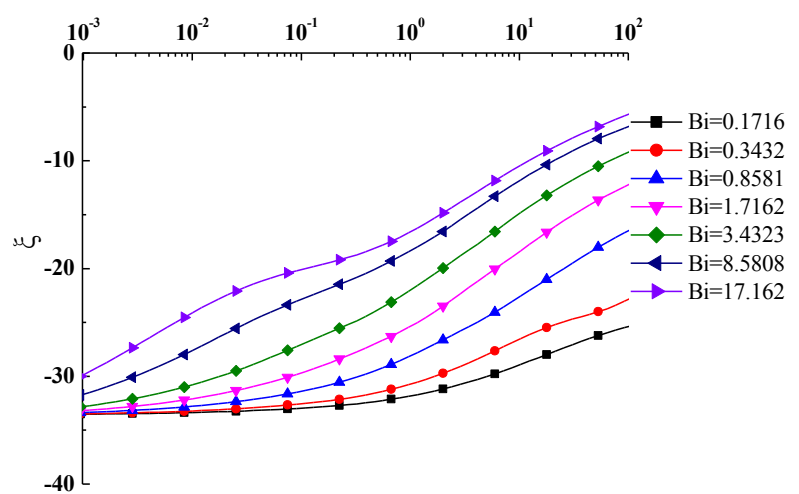

Fo

(c) Equal perimeter approach

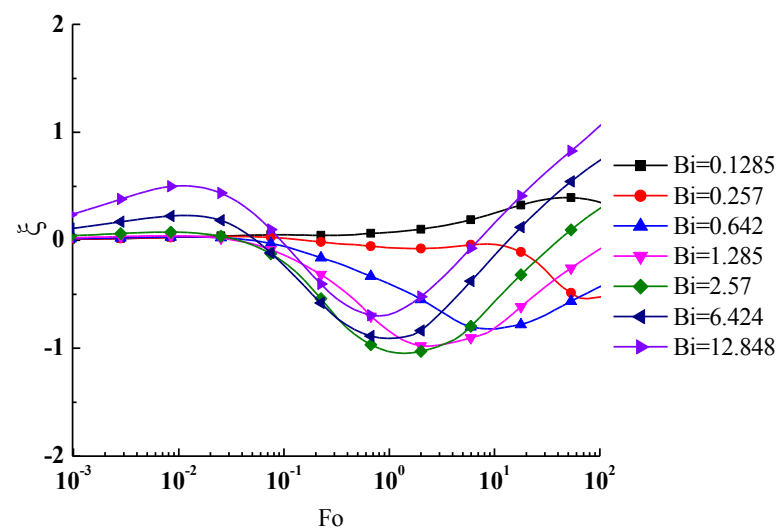

Fig. 4. Relative error of the heat dissipation of the trapezoid roadway under three circumstances

In Figure 4, when the equal area method is used, the relative error decreases with the change in time. The maximum relative error of the trapezoid roadway is $22 \%$ under any $\mathrm{Bi}$. The relative error decreases to $10 \%$ when $\mathrm{Fo}=$ 100 and $\mathrm{Bi}=10$, as shown in Figure 4(a). When the equal hydraulic diameter method is used, the maximum negative deviation is $35 \%$ and the minimum negative deviation is $10 \%$, with $\mathrm{Bi}=0.1716$. However, the variation range of the absolute value of the relative error is $0 \%$ to $1 \%$ for the equal perimeter method. This result is evidently less than those of the other methods.

\subsection{Analysis of Relative Errors of Rectangular Roadway}

(a) Equal area approach

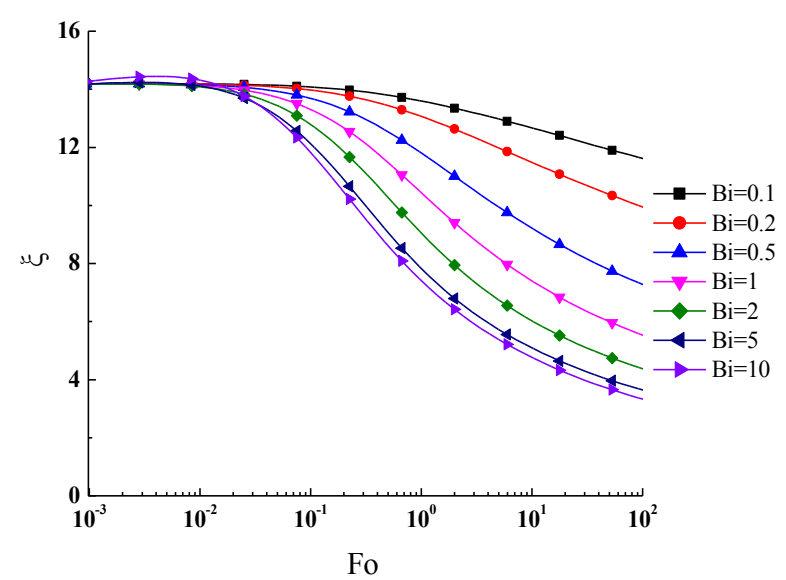

(b) Equal hydraulic diameter approach

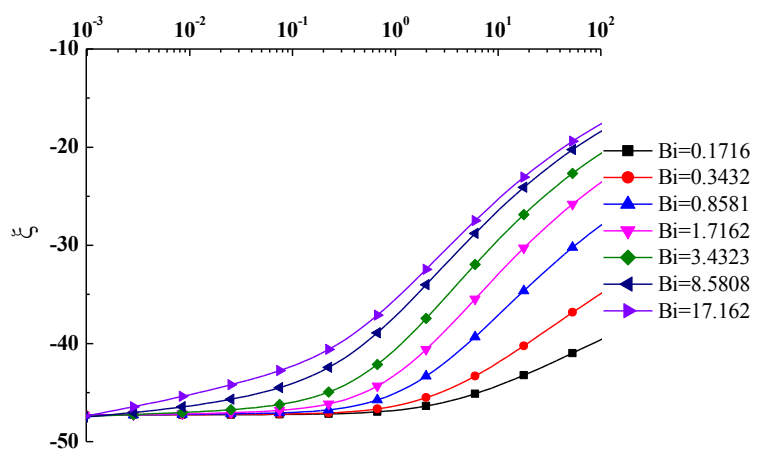

Fo

(c) Equal perimeter approach

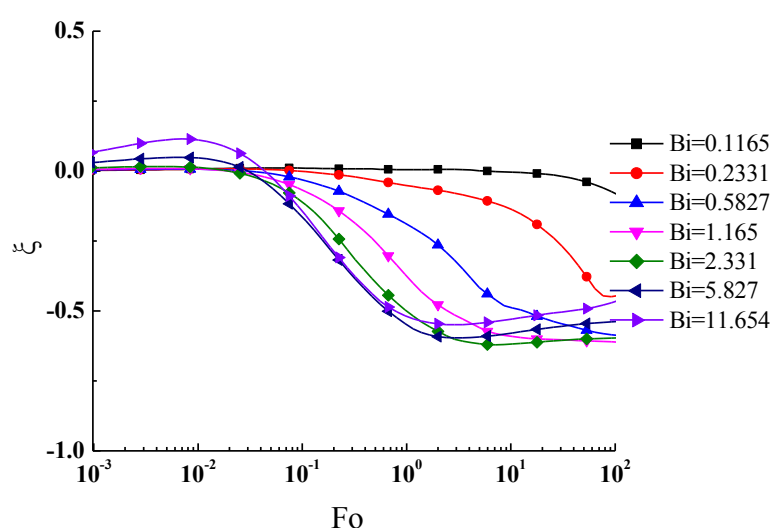

Fig. 5. Relative error of the heat dissipation of the rectangular roadway under three circumstances

Fig. 5 shows that for the rectangular roadway, the range of the relative error of heat dissipation is $10 \%$ to $20 \%$ for the equal area method, $20 \%$ to $50 \%$ for the equal hydraulic diameter approach, and $0 \%$ to $0.6 \%$ for the equal perimeter 
method under any $\mathrm{Bi}$. The result of the equal perimeter method is evidently less than those of the other methods.

\subsection{Analysis of Relative Errors of Arch Roadway}

(a) Equal area approach

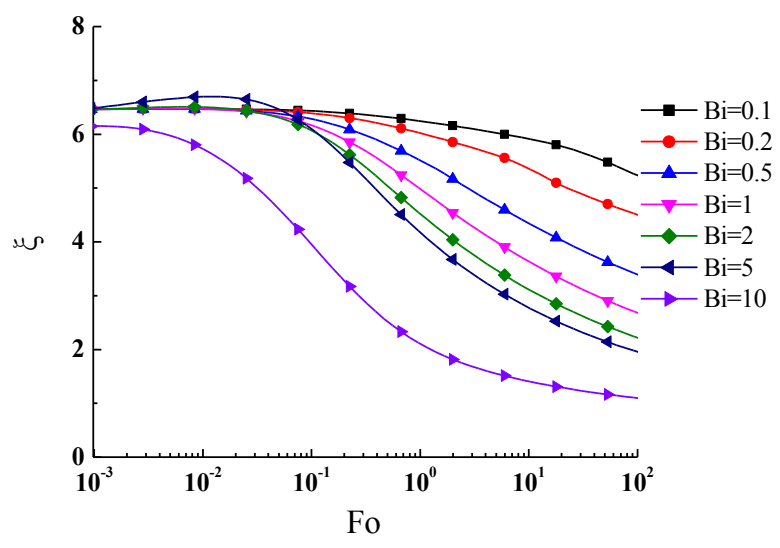

(b) Equal hydraulic diameter approach

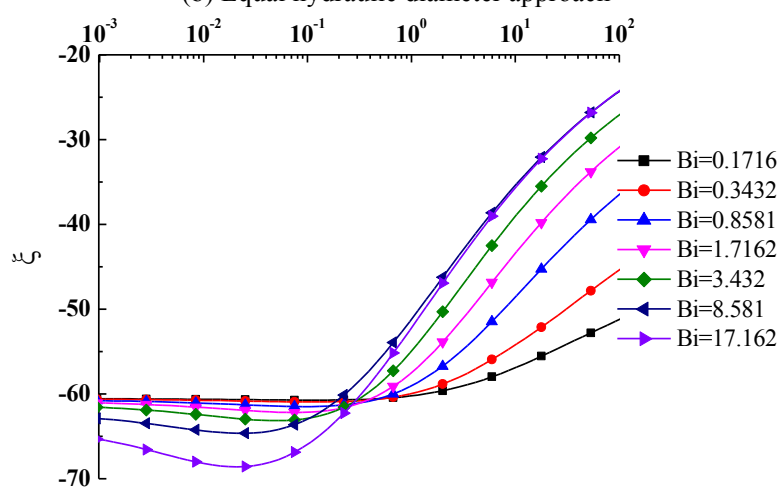

Fo

(c) Equal perimeter approach

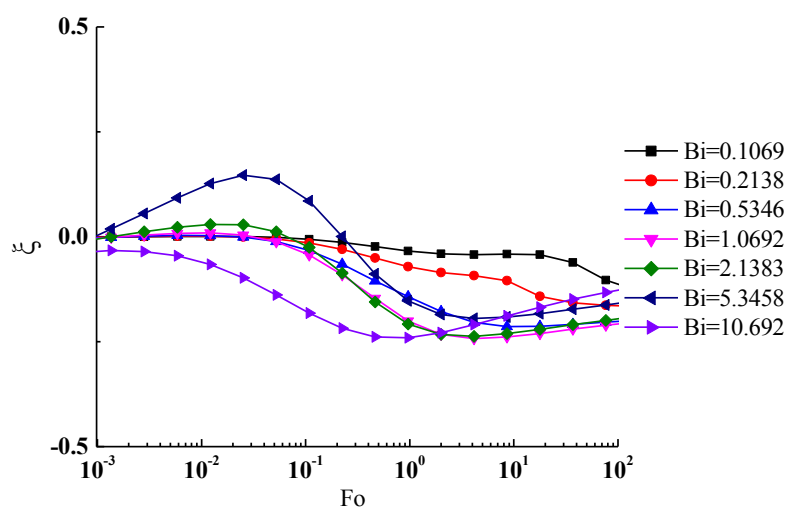

Fig. 6. Relative error of the heat dissipation of the arch roadway under three circumstances

In Figure 6, the relative error of the heat dissipation of the arch roadway has the same variation feature as the other two roadways. When Fo ranges from 0.0001 to 100 , the relative error of the equal area method ranges from $6.5 \%$ to $2 \%$ and the relative error of the equal hydraulic diameter method ranges from $20 \%$ to $50 \%$. However, the relative error of the equal perimeter method ranges from $0 \%$ to $0.25 \%$. Thus, the equal perimeter approach is evidently superior to the other methods, too.

For the three roadway surrounding rocks, the relative error of heat dissipation of the equal perimeter method is evidently smaller than those of the other methods. Thus, preferring the equal perimeter method when calculating heat dissipation capability is reasonable.

The equivalent radius is essentially an equivalent measure, but not an equal value. Objectively, the relative errors are inevitable when we adopt the equivalent radius to calculate the heat dissipation problem. However, in practice, we should select a relatively accurate approach to calculate the heat dissipation capability to obtain more precise results. For the equivalent radius problem, employing the equal perimeter method in engineering applications is evidently beneficial.

\section{Conclusion}

A model of the unsteady state temperature field of surrounding rocks is established. The dimensionless criterion is introduced to analyze the governing equations of surrounding rocks in cylindrical and rectangular coordinate systems. The calculation methods of heat dissipation capability and heat transfer resistance of surrounding rocks are derived from differential equations. The generality and difference of surrounding rock heat dissipation of three equivalent circular roadway methods are compared and analyzed. The equal perimeter roadway method is determined to be the best approach. In this study, the deviations of heat dissipation of surrounding rock between circular and non-circular roadways with trapezoidal, rectangular, and arch cross-sectional forms are investigated. The results show that the deviation of the equal perimeter circular roadway method is the lowest and the calculation approach of the surrounding rock heat dissipation of noncircular roadways with equal perimeter is the best.

In practical applications, we can generally calculate Fo and $\mathrm{Bi}$ using the optimal equivalent radius, called the equal perimeter, based on the initial conditions. Then, the unstable heat transfer criterion can be confirmed based on the results shown in Figure 1. Finally, the heat dissipation capability of roadway surrounding rocks can be determined using Eq. 10. This study on equivalent radius is standardized, which provides a theoretical basis for engineering applications.

\section{Acknowledgements}

This work was supported by the National Natural Science Foundation of China under the project No. 51174211.

\section{References}

1. Bluhm, S. J., Biffi, M., and Wilson, R. B., "Optimized Cooling Systems for Mining at Extreme Depths", Cim Bulletin, 93(1), 2000, pp. $146-150$

2. Gu, B. S., "Countermeasures against high temperatures in a tunnel and the corresponding ventilation design", Modern Tunnelling Technology, 44(2), 2007, pp. 66-71.
3. He, M. C., and Guo, P. Y., "Deep rock mass thermodynamic effect and temperature control measures", Chinese Journal of Rock Mechanics and Engineering, 32(12), 2013, pp. 2377-2393.

4. Yuan, L., "Theoretical analysis and practical application of coal mine cooling in Huainan mining area", Journal of Mining and Safety Engineering, 34(3), 2007, pp. 298-301. 
5. Yang, X. J., Han, Q. Y., Pang, J. W., Shi, X. W., Hou, D. G. and Liu, C., "Progress of heat-hazard treatment in deep mines", Mining Science and Technology (China), 21(2), 2011, pp. 295-299.

6. Gideon E. P., Leon, L. and Edward, H. M., "Case study: The effects of a variable flow energy saving strategy on a deep-mine cooling system", Apply Energy, 102, 2013, pp. 700-709.

7. Inyang, H. I., and Daniels, J. L., "Environmental issues from coal mining and their solutions", Mining Science and Technology, 20(2), 2010, pp. 215-223.

8. Ji, J. H., Liao, Q., Hu, Q. T., Chu, Z. X., Zhang, X. J., and Gong, L. P., "Heat transfer characteristics of driving face in heat harm mine", Journal of China Coal Society, 39(4), 2014, pp. 692-698.

9. He, G. J., Ruan, G. Q., and Yang, Z., "Research and application on preventive measures against heat disaster in Zhaolou Coal Mine", Journal of China Coal Society, 36(1), 2011, pp. 101-104.

10. Wang, Y. J., "Heat and mass transfer of surrounding rock and airflow for deep thermal environment", China University of Mining and Technology, Xuzhou, 2010, pp. 41-52.

11. Yu, H. C., Deng, X., and Chen, B. W., "Mine geothermal and heat harm management", Coal Industry Press, Beijing, 1991, pp. $292-$ 293.

12. Щсрбаиъ. АП., “The mine cooling guide", Coal Industry Publishing House, Beijing, 1982, pp. 80-90.

13. Cen, Y. Q., Hu, C. S., and Hou, Q. Z., "Investigation into unsteady heat transfer coefficient Kut between the surrounding rock of mine wells or lanes and airflow", Journal of Liaoning Technical University, 6(3), 1987, pp. 105-114.

14. Sun, P. D., and Zhu, C. Q., "Study on the geothermal fields of country rock on deep tunnels", Journal of China University of Mining \& Technology, 19(2), 1989, pp. 27-34.

15. Starfield, A. M., and Bleloch, A. L., "New method for the computation of heat and moisture transfer in a partly wet airway", Journal of the South Africa Institute of Mining and Metallurgy, 83(11), 1983, pp. 263-269.

16. Yakovenko, A. K., and Averin, G. V., "Determination of the heattransfer coefficient for a rock mass with small Fourier numbers" Fiziko-teknicheskle Problemy Razrabotkl Poleznykh Iskopaemykh, 1(20), 1984, pp. 52-56.

17. Gao, J. L., Xu, W., and Zhang, X. B., "Treatment of water evaporative during calculation of temperature and humidity of airflow caused by heat release from surrounding rock", Journal of China coal Society, 35(1), 2010, pp. 951-955.

18. Zhang, X. F., Yu, W. B., Wang, C. and Liu, Z. Q., "Threedimensional nonlinear analysis of coupled problem of heat transfer in the surrounding rock and heat convection between the air and the surrounding rock in the Fenghou mountain tunnel", Chinese Journal of Geotechnical Engineering, 27(12), 2005, pp. 1413-1420.

19. Wu, Q., Qin, Y. P., Guo, L., and Tan, K., "Analysis of unsteady temperature field in wall rock by finite element method", Journal of Liaoning Technical University, 21(5), 2002, pp. 605-608.

20. Wu, Q., Qin, Y. P., Guo, L., and Wu, Q. Y., "Calculation of the Heat Emitting from the Wall Rock at Drifting Face with Finite Element Method", China Safety Science Journal, 12(6), 2002, pp. 33-36.

21. Lowndes, I. S., Crossley, A. J., and Yang, Z. Y., "The ventilation and climate modelling of rapid development tunnel drivages", Tunnelling and Underground Space Technology, 19(2), 2004, pp. 139-150.

22. Zhang, Y., Wan, Z. J., Zhou, C. B., and Cheng, J. Y., "Modeling experimental method of unsteady heat conduction in surrounding rock of roadways or tunnels", Journal of Mining \& Safety Engineering, 31(3), 2014, pp. 441-446.

23. Danko, G., and Bahrami, D., "Application of MULTIFLUX for air, heat and moisture flow simulations. Wallace", 12th U. S. North American Mine Ventilation Symposium. Nevada University Press, Nevada, 2008, pp. 267-274.

24. Qin, Y. P., Song, H. T., Wu, J. S., and Dong, Z. Y., "Numerical analysis of temperature field of surrounding rock under periodic boundary using Finite Volume Method”, Journal of China Coal Society, 40(7), 2015, pp. 1541-1549.

25. Qin, Y. P., Wang, J., Hao, Y. J., Liu, W., and Kong, S., "Dimensionless Analysis for Heat Dissipation from Surrounding Rock in roadway", Mining and Metallurgical Engineering, 33(3), 2013, pp. 5-12.

26. Wang, Y. H., "Study on simulation of Spontaneous Combustion in Goaf on the Moving Coordinate with Finite Volume Method", China university of Mining and Technology, Beijing, 2009, pp. 921. 